\title{
Nuclear Astrophysics with RIBs at Oak Ridge National LaboratoryRECEIVED
}

\section{CONF-981122-}

\author{
D. W. Bardayan
}

\author{
Physics Division, Oak Ridge National Laboratory, Oak Ridge, Tennessee 37831 \\ and \\ A. W. Wright Nuclear Structure Laboratory, Yale University, New Haven, Connecticut 06511
}

\begin{abstract}
The Daresbury Recoil Separator (DRS) and Silicon Detector Array (SIDAR) have been installed at Oak Ridge National Laboratory (ORNL) to perform measurements of reaction cross sections of astrophysical interest using radioactive ion beams (RIBs). For example radioactive ${ }^{17} \mathrm{~F}$ beams will be used to determine the ${ }^{14} \mathrm{O}(\alpha, p){ }^{17} \mathrm{~F}$ and ${ }^{17} \mathrm{~F}(\mathrm{p}, \gamma)^{18} \mathrm{Ne}$ stellar reaction rates - both of which are important reactions in the Hot-CNO cycle. The first reactions studied will be ${ }^{1} \mathrm{H}\left({ }^{17} \mathrm{~F}, \mathrm{p}\right){ }^{17} \mathrm{~F}$ and ${ }^{1} \mathrm{H}\left({ }^{17} \mathrm{~F}, \alpha\right){ }^{14} \mathrm{O}$. These experiments will require ${ }^{17} \mathrm{~F}$ beams with intensities of $10^{4}-10^{6}$ ions per second in conjunction with the SDAR. The ${ }^{1} \mathrm{H}\left({ }^{17} \mathrm{~F}, \mathrm{p}\right){ }^{17} \mathrm{~F}$ reaction will be used to probe resonances in ${ }^{18} \mathrm{Ne}$ which contribute to the ${ }^{17} \mathrm{~F}(\mathrm{p}, \gamma)^{18} \mathrm{Ne}$ stellar reaction rate, while ${ }^{1} \mathrm{H}\left({ }^{17} \mathrm{~F}, \alpha\right){ }^{14} \mathrm{O}$ will be used to determine the stellar reaction rate of the inverse reaction ${ }^{14} \mathrm{O}(\alpha, p){ }^{17} \mathrm{~F}$. In preparation for these experiments, measurements have been made of the ${ }^{1} \mathrm{H}\left({ }^{17} \mathrm{O}, \mathrm{p}\right){ }^{17} \mathrm{O}$ and ${ }^{1} \mathrm{H}\left({ }^{17} \mathrm{O}, \alpha\right){ }^{14} \mathrm{~N}$ reaction cross sections. When higher beam currents of ${ }^{17} \mathrm{~F}$ become available, a direct measurement of the ${ }^{1} \mathrm{H}\left({ }^{17} \mathrm{~F},{ }^{18} \mathrm{Ne}\right)$ resonance strength will be made using the DRS. To test the performance of the DRS for measuring capture reaction cross sections, the well-known ${ }^{1} \mathrm{H}\left({ }^{12} \mathrm{C},{ }^{13} \mathrm{~N}\right)$ cross section has been measured at an energy similar to those proposed for radioactive beam experiments. Results from these stable beam experiments are discussed.
\end{abstract}

\section{INTRODUCTION}

There are a number of astrophysical events during which hydrogen serves as fuel for $(\mathrm{p}, \gamma)$ fusion reactions under non-hydrostatic equilibrium conditions. These explosive hydrogen burning events, which include novae and $\mathrm{X}$-ray bursts, are among the most energetic explosions $\left(\sim 10^{38-45}\right.$ ergs $)$ known in the universe. They are characterized by extremely high temperatures $\left(10^{8-9} \mathrm{~K}\right)$ and densities $\left(10^{3-5} \mathrm{~g} / \mathrm{cm}^{3}\right)$, conditions which cause $(\mathrm{p}, \gamma)$ reactions to rapidly (on timescales of $\mathrm{ns}-\mathrm{min}$ ) produce nuclei on the proton-rich side of the valley of stability. Any such nuclei produced with beta-decay half-lives longer than, or comparable to, the mean time between fusion events will become targets for subsequent nuclear reactions. Sequences of $(p, \gamma)$ reactions on proton-rich radioactive nuclei can therefore occur during these explosions (1), and the observable ashes of such nuclear burning sequences are an important probe of the conditions in these events.

The Hot-CNO (HCNO) cycle $\left({ }^{12} \mathrm{C}(\mathrm{p}, \gamma)^{13} \mathrm{~N}(\mathrm{p}, \gamma)^{14} \mathrm{O}\left(\mathrm{e}^{+} \mathrm{v}_{\mathrm{e}}\right)\right.$ $\left.{ }^{14} \mathrm{~N}(\mathrm{p}, \gamma)^{15} \mathrm{O}\left(\mathrm{e}^{+} v_{\mathrm{e}}\right)^{15} \mathrm{~N}(\mathrm{p}, \alpha)^{12} \mathrm{C}\right)$ is a primary reaction chain through which explosive hydrogen burning occurs. The energy generation rate of this sequence is limited by the beta-decay lifetimes of ${ }^{14} \mathrm{O}$ and ${ }^{15} \mathrm{O}$ at moderately high temperatures. When the stellar temperatures are high enough (approximately 300 million degrees or higher), the beta-decay of ${ }^{14} \mathrm{O}$ can be bypassed by the ${ }^{14} \mathrm{O}(\alpha, \mathrm{p}){ }^{17} \mathrm{~F}$ reaction, and the reaction sequence ${ }^{14} \mathrm{O}(\alpha, \mathrm{p}){ }^{17} \mathrm{~F}(\mathrm{p}, \gamma)^{18} \mathrm{Ne}\left(\mathrm{e}^{+} v_{\mathrm{e}}\right)$ $\left.{ }^{18} \mathrm{~F}(p, \alpha)\right)^{15} \mathrm{O}$ can increase the energy generation rate and alter the abundances of the $\mathrm{CNO}$ nuclides. The reaction sequence ${ }^{16} \mathrm{O}(\mathrm{p}, \gamma){ }^{17} \mathrm{~F}(\mathrm{p}, \gamma){ }^{18} \mathrm{Ne}\left(\mathrm{e}^{+} v_{\mathrm{e}}\right)^{18} \mathrm{~F}(\mathrm{p}, \alpha){ }^{15} \mathrm{O}$ can alter the CNO nuclide abundances as well, while the sequence ${ }^{14} \mathrm{O}(\alpha, \mathrm{p}){ }^{17} \mathrm{~F}(\mathrm{p}, \gamma){ }^{18} \mathrm{Ne}\left(\mathrm{e}^{+} v_{\mathrm{e}}\right){ }^{18} \mathrm{~F}(\mathrm{p}, \gamma){ }^{19} \mathrm{Ne}(\mathrm{p}, \gamma){ }^{20} \mathrm{Na}$ can provide a path from the HCNO cycle into the rapid proton (rp) capture process. The energy generation rate in the rpprocess can be two orders of magnitude larger than the HCNO cycle (1), and at temperatures over one billion degrees, elements more massive than $\mathrm{Fe}$ can possibly be formed (2). In order to understand these cataclysmic stellar events, we must know the ${ }^{17} \mathrm{~F}(\mathrm{p}, \gamma){ }^{18} \mathrm{Ne}$ and ${ }^{14} \mathrm{O}(\alpha, \mathrm{p}){ }^{17} \mathrm{~F}$ stellar reaction rates.

Comparisons of astrophysical models with the latest observations require measurements of the important reactions involving radioactive isotopes. By producing highquality, intense beams of the radioactive ions involved in explosive nucleosynthesis, the Holifield Radioactive Ion Beam Facility (HRIBF) at ORNL $(3,4)$ has the potential to significantly improve the understanding of these spectacular stellar explosions. Radioactive ion beams are produced at HRIBF by an ISOL-type target/ion source $(5,6)$. A high temperature $\left(1100-2200^{\circ} \mathrm{C}\right)$ refractory target is bombarded by a $0.5 \mathrm{~kW}$ light ion ( $\mathrm{p}, \mathrm{d},{ }^{3} \mathrm{He}$, or ${ }^{4} \mathrm{He}$ ) beam from the $\mathrm{K}=105$ Oak Ridge Isochronous Cyclotron (ORIC). The radioactive reaction products then diffuse out of the hot target material and through a short $(10 \mathrm{~cm})$ transfer tube to a modular ion source, where they are ionized and ex- 


\section{DISCLAIMER}

This report was prepared as an account of work sponsored by an agency of the United States Government. Neither the United States Government nor any agency thereof, nor any of their employees, makes any warranty, express or implied, or assumes any legal liability or responsibility for the accuracy, completeness, or usefulness of any information, apparatus, product, or process disclosed, or represents that its use would not infringe privately owned rights. Reference herein to any specific commercial product, process, or service by trade name, trademark, manufacturer, or otherwise does not necessarily constitute or imply its endorsement, recommendation, or favoring by the United States Government or any agency thereof. The views and opinions of authors expressed herein do not necessarily state or reflect those of the United States Government or any agency thereof. 


\section{DISCLAIMER}

Portions of this document may be illegible in electronic image products. Images are produced from the best available original document. 
tracted. Fibrous $\mathrm{Al}_{2} \mathrm{O}_{3}$ targets (7) are being used to produce ${ }^{17} \mathrm{~F}$ via the ${ }^{16} \mathrm{O}(\mathrm{d}, \mathrm{n}){ }^{17} \mathrm{~F}$ reactions at $30 \mathrm{MeV}$. Once produced the radioactive ions are charge-exchanged (if they are positive ions) and then undergo two stages of mass analysis (with $\Delta m / m<10^{-4}$ ) before their injection into the 25-MV tandem accelerator, subsequent acceleration, and delivery to the experimental areas.

\section{THE ${ }^{17} F(p, y)^{18}$ Ne REACTION}

The ${ }^{17} \mathrm{~F}(\mathrm{p}, \gamma){ }^{18} \mathrm{Ne}$ stellar reaction rate at temperatures characteristic of stellar explosions is thought to be dominated by resonances in ${ }^{18} \mathrm{Ne}$ at excitation energies of 4.520 , 4.561 , and $4.589 \mathrm{MeV}$. The $4.561 \mathrm{MeV}$ resonance, however, has only been observed in a measurement of the ${ }^{16} \mathrm{O}\left({ }^{3} \mathrm{He}, \mathrm{n}\right){ }^{18} \mathrm{Ne}$ reaction (8), but it was not observed in measurements of the ${ }^{20} \mathrm{Ne}(\mathrm{p}, \mathrm{t})^{18} \mathrm{Ne}$ and ${ }^{12} \mathrm{C}\left({ }^{12} \mathrm{C},{ }^{6} \mathrm{He}\right){ }^{18} \mathrm{Ne}$ reactions $(9,10)$. From comparison to the isobaric analog nucleus ${ }^{18} \mathrm{O}$, this resonance may have $\mathrm{J}^{\pi}=3^{+}$, which potentially makes it a strong $l=0$ transition in the ${ }^{17} \mathrm{~F}(\mathrm{p}, \gamma)^{18} \mathrm{Ne}$ reaction. The ${ }^{17} \mathrm{~F}(\mathrm{p}, \gamma){ }^{18} \mathrm{Ne}$ capture reaction itself - with a cross section $\approx 2.5 \mu \mathrm{b}$ - is too weak to be used to search for the resonance. We, therefore, plan to first measure the ${ }^{1} \mathrm{H}\left({ }^{17} \mathrm{~F}, \mathrm{p}\right){ }^{17} \mathrm{~F}$ excitation function at the HRIBF to confirm the existence of this state and measure its properties: resonance energy, width, spin, and parity. Since the cross section for this reaction is large $(\sim 1$ barn $)$, this measurement can be performed with relatively low ${ }^{17} \mathrm{~F}$ beam currents $\left(10^{4}{ }^{17} \mathrm{~F} / \mathrm{s}\right.$ with a $50 \mu \mathrm{g} / \mathrm{cm}^{2} \mathrm{CH}_{2}$ target). Once the resonance is found and higher beam currents of ${ }^{17} \mathrm{~F}$ become available, a direct measurement of the resonant ${ }^{17} \mathrm{~F}(\mathrm{p}, \gamma)^{18} \mathrm{Ne}$ cross section will be made using the Daresbury Recoil Separator (DRS) now located at ORNL. An intensity of approximately $6 \times 10^{7}(0.01 \mathrm{pnA})$ would be required to make a $10 \%$ measurement in 25 days at the dominant resonance.

The elastic scattering excitation function of ${ }^{17} \mathrm{~F}(\mathrm{p}, \mathrm{p}){ }^{17} \mathrm{~F}$ will be measured with $a{ }^{17} \mathrm{~F}$ beam and a polypropylene $\left(\mathrm{CH}_{2}\right)_{\mathrm{n}}$ transmission target in inverse kinematics (i.e., $\left.{ }^{1} \mathrm{H}\left({ }^{17} \mathrm{~F}, \mathrm{p}\right){ }^{17} \mathrm{~F}\right)$. The scattered protons will be detected in an annular array of single-sided silicon strip detectors in the target chamber downstream of the target location. This silicon detector array (SIDAR) is comprised of $128 \mathrm{seg}$ ments with 16 radial (from 5 to $13 \mathrm{~cm}$ ) and 8 azimuthal divisions, similar to the LEDA array used at Louvain-laNeuve (11). The array subtends 15 to 35 degrees in the lab, allowing detection of the forward-focused protons while passing the ${ }^{17} \mathrm{~F}$ beam out of the target chamber. The SIDAR has the advantages of large solid angle coverage and simultaneous measurements of the particle energy and angular distribution. With a beam current of $10^{4}{ }^{17} \mathrm{~F} / \mathrm{s}$ and a $\mathrm{CH}_{2}$ foil of thickness $50 \mu \mathrm{g} / \mathrm{cm}^{2}$, an average of 300 protons per hour will be detected in the SIDAR. This will allow $3 \%$ statistics to be obtained in 4 hours at each beam energy, and several beam energies between 10 and $13 \mathrm{MeV}$ would be used to map out the excitation function. From

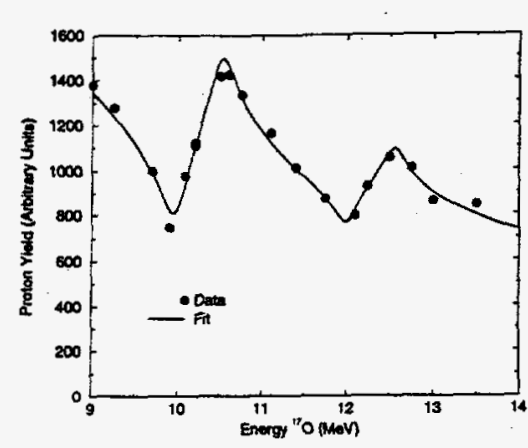

FIGURE 1. The ${ }^{1} \mathrm{H}\left({ }^{17} \mathrm{O}, \mathrm{p}\right){ }^{17} \mathrm{O}$ excitation function.

the excitation function, the resonance energy and width of the state can be extracted. From the proton angular distribution measured while on resonance, the spin and parity of the state can be deduced.

In preparation for this experiment, a measurement of the ${ }^{1} \mathrm{H}\left({ }^{17} \mathrm{O}, \mathrm{p}\right){ }^{17} \mathrm{O}$ excitation function has been performed. The energy range of the ${ }^{17} \mathrm{O}$ beam was picked to populate the isobaric analog in ${ }^{18} \mathrm{~F}$ of the $3^{+}$state sought in ${ }^{18} \mathrm{Ne}$. We also included in our measurement a nearby $2^{+}$state in ${ }^{18} \mathrm{~F}$. This allowed us to examine the sensitivity of the angular distribution measurement to the spin and parity of the state that we are populating. Proton yields were measured for 19. beam energies from 9 to $13.5 \mathrm{MeV}$ over a period of 4 days with ${ }^{17} \mathrm{O}$ beam currents of about $5 \times 10^{6}{ }^{17} \mathrm{O} / \mathrm{s}$. The normalized proton yields are plotted in Fig. 1 along with a fit to the data. The fit was made using a Breit-Wigner formalism (12), and the fit results are given in Table 1. The proton angular distributions are plotted in Fig. 2 and clearly show our sensitivity to the spins and parities of the states involved. We get a much better fit to the $10.08 \mathrm{MeV}$ angular distribution data if we assume a $3^{+}$state in the fit; while the $12.24 \mathrm{MeV}$ angular distribution data is fit much better by a $2^{+}$angular distribution. This agrees with the known spins and parities of these states (13).

A direct measurement of the ${ }^{1} \mathrm{H}\left({ }^{17} \mathrm{~F},{ }^{18} \mathrm{Ne}\right)$ reaction cross section will be made using the DRS (14). The ${ }^{18} \mathrm{Ne}$ recoils will be detected at the DRS focal plane by a positionsensitive carbon-foil microchannel plate and a $\triangle E-E$ gas ionization counter. This method of direct recoil detection in inverse kinematics has several advantages over traditional capture $\gamma$-ray detection techniques (15). A high detection efficiency is possible because all recoils are within a cone of half-angle $0.5^{\circ}$ with respect to the beam direction, and the signal-to-noise ratio is improved because the detectors are located far from the target. Other detection methods also become possible such as delayed activity detection

TABLE 1. The $\left.{ }^{1} \mathrm{H}^{17} \mathrm{O}, \mathrm{p}\right)^{17} \mathrm{O}$ Fit Results.

\begin{tabular}{cccc}
\hline$J^{\pi}$ & & Fit Results & Accepted (13) \\
\hline $3^{+}$ & $E_{x}(\mathrm{MeV})$ & $6.1605 \pm 0.0009$ & $6.1632 \pm 0.0009$ \\
$3^{+}$ & $\Gamma(\mathrm{keV})$ & $13.8 \pm 0.6$ & $14.0 \pm 0.5$ \\
$2^{+}$ & $E_{x}(\mathrm{MeV})$ & $6.2741 \pm 0.0014$ & $6.2832 \pm 0.0009$ \\
$2^{+}$ & $\Gamma(\mathrm{keV})$ & $11.4 \pm 0.9$ & $10.0 \pm 0.5$ \\
\hline
\end{tabular}




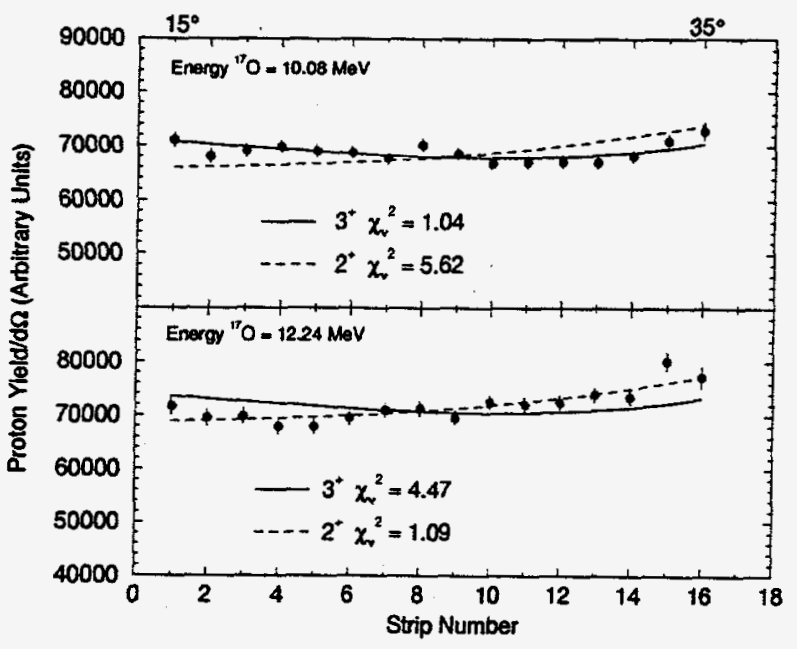

FIGURE 2. The ${ }^{1} \mathrm{H}\left({ }^{17} \mathrm{O}, \mathrm{p}\right){ }^{17} \mathrm{O}$ angular distributions at the resonance energies. The solid line is a fit assuming that the state is a $3^{+}$resonance. The dashed line is a fit assuming that the state is a $2^{+}$resonance. The reduced $\chi^{2}$ of the fits are shown.

and recoil- $\gamma$ coincidences. The recoil detection approach is, however, challenging because: the separator must be located along the beam axis and accepts all of the beam particles; the recoils are only $10^{-10}$ to $10^{-12}$ times as intense as the projectiles; and the projectiles and recoils are similar, having nearly identical momentum and differing in velocity and mass by only a few percent. The recoil separator must, therefore, be optimized to collect the ions of interest while simultaneously suppressing the transmission of unwanted scattered beam projectiles. The focal plane detectors must also be capable of distinguishing the recoils from the projectiles, which is quite challenging in view of the low energy $(0.4-2.0 \mathrm{MeV} / \mathrm{amu})$ and low mass of the particles to be identified.

To test the performance of the DRS for measuring capture reaction cross sections, a measurement of the wellknown ${ }^{1} \mathrm{H}\left({ }^{12} \mathrm{C},{ }^{13} \mathrm{~N}\right)$ cross section has been made. A 0.666 $\mathrm{MeV} / \mathrm{amu}{ }^{12} \mathrm{C}$ beam was used to bombard a $\mathrm{CH}_{2}$ target, and ${ }^{13} \mathrm{~N}$ recoils were collected at the focal plane of the DRS for 12 hours. The ionization counter spectrum for this capture reaction measurement is shown in Fig. 3. The suppression of scattered beam particles - defined by the ratio of beam particles incident on target to those reaching the focal plane - was $3 \times 10^{-11}$, which is within the range needed $\left(10^{-10}\right.$ to $\left.10^{-12}\right)$ for capture reaction measurements. This rejection value is consistent with previous measurements that utilized elastic scattering reactions (e.g. $\left.{ }^{12} \mathrm{C}\left({ }^{14} \mathrm{~N},{ }^{12} \mathrm{C}\right){ }^{14} \mathrm{~N}\right)(16)$. Furthermore, the gas ionization counter cleanly separated the ${ }^{13} \mathrm{~N}$ recoils from the scattered ${ }^{12} \mathrm{C}$ projectiles. The combined projectile rejection of the DRS and the focal plane detector was, therefore, beyond that needed for capture reaction measurements. The DRS transmission, however, for the recoils was $7 \%$, a factor of 7 lower than expected. Since the $\mathrm{CH}_{2}$ targets could only withstand $0.5 \mathrm{pnA}$ of beam, the ${ }^{13} \mathrm{~N}$ count rate was only a

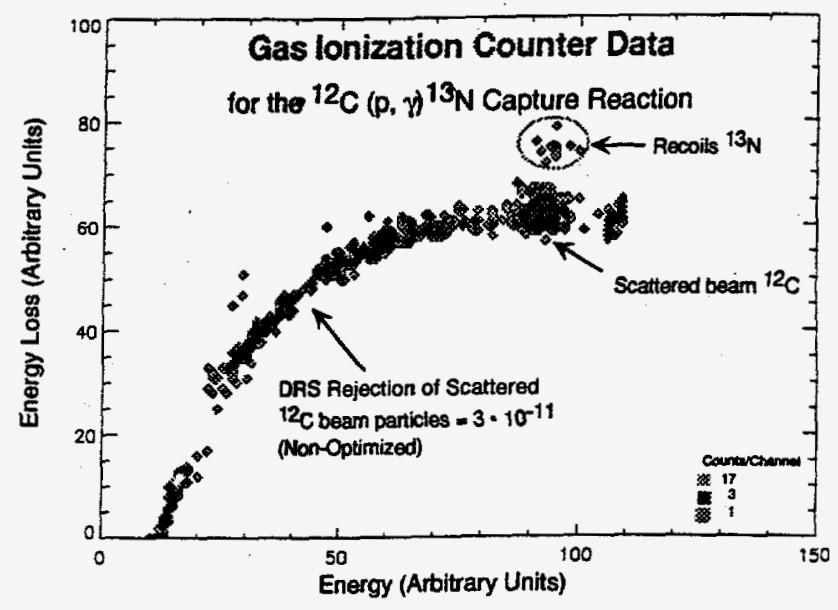

FIGURE 3. Particle identification in the DRS ionization counter.

few counts per hour which wàs too low to be used to optimize the DRS settings. We have found in subsequent tests with scattering and fusion-evaporation reactions that the optical elements of the DRS were not appropriately tuned during this measurement and that the dispersions of the different sections of the DRS were not matched properly. This resulted in the ${ }^{13} \mathrm{~N}$ recoils not being focused at the spectrometer focal plane and a reduction in the observed transmission. Optimization of the DRS using fusionevaporation and scattering reactions is in progress, and measurements of capture reactions with stable beams are planned for the future.

\section{THE ${ }^{14} O(\alpha, p){ }^{17}$ F REACTION}

The rate for the ${ }^{14} \mathrm{O}(\alpha, \mathrm{p}){ }^{17} \mathrm{~F}$ reaction is also quite uncertain. Stable beam spectroscopy measurements indicate that a $1^{1}$ state at $\mathrm{E}_{\mathrm{x}}=6.150 \mathrm{MeV}$ in ${ }^{18} \mathrm{Ne}$ provides the dominant resonant contribution to the ${ }^{14} \mathrm{O}(\alpha, p){ }^{17} \mathrm{~F}$ reaction rate at temperatures less than $10^{9} \mathrm{~K}$. At these temperatures, the rate depends upon the unknown spectroscopic factors (total and partial-alpha widths) of the $6.150 \mathrm{MeV}$ state, as well as upon the $l=1$ direct reaction component and the interference between them (9). Higher energy states, including those observed at $6.29,7.05,7.12$, and $7.35 \mathrm{MeV}$, are expected to make a significant contribution to the reaction rate at temperatures greater than $10^{9} \mathrm{~K}$.

A measurement of the ${ }^{1} \mathrm{H}\left({ }^{17} \mathrm{~F}, \alpha\right){ }^{14} \mathrm{O}$ reaction will be made to determine spectroscopic properties of resonances at $E_{x}=6.0-7.5 \mathrm{MeV}$ in ${ }^{18} \mathrm{Ne} . \mathrm{A}{ }^{17} \mathrm{~F}$ beam with energies between 39 and $64 \mathrm{MeV}$ will be used with a transmission polypropylene $\mathrm{CH}_{2}$ target. The recoil alpha particles will be detected in the SIDAR which will be run in "telescope" mode (i.e. a $100-\mu \mathrm{m}$-thick detector backed by a $500-\mu \mathrm{m}$ thick detector) to discriminate between the alpha recoils and scattered protons and projectiles. The SIDAR will subtend angles 10 to $25^{\circ}$ in the lab. Additionally, the ${ }^{14} \mathrm{O}$ recoils will be detected by a smaller annular silicon detector which will be placed behind the SIDAR and subtend angles 3.2 to $6.5^{\circ}$ in the lab. This "mini" detector is also 


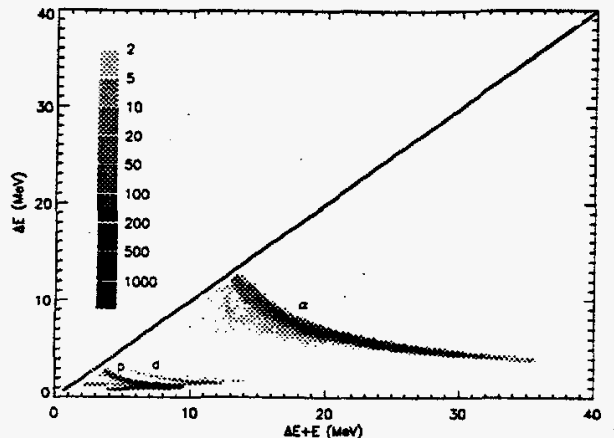

FIGURE 4. Particle identification from the ${ }^{1} \mathrm{H}\left({ }^{17} \mathrm{O}, \alpha\right){ }^{14} \mathrm{~N}$ reaction using the SIDAR.

highly segmented (16 radial and 4 azimuthal divisions) and, therefore, can handle the high counting rates from elastic scattering experienced at such forward angles without significant pileup.

In preparation for this experiment, we have performed a measurement of the ${ }^{1} \mathrm{H}\left({ }^{17} \mathrm{O}, \alpha\right){ }^{14} \mathrm{~N}$ reaction. A $40 \mathrm{MeV}{ }^{17} \mathrm{O}$ beam $\left(5 \times 10^{6}{ }^{17} \mathrm{O} / \mathrm{s}\right)$ was used to bombard a $100 \mu \mathrm{g} / \mathrm{cm}^{2}$ $\mathrm{CH}_{2}$ target for a period of 3 days. The SIDAR particle identification is shown in Fig. 4 where the alpha recoils are cleanly separated from the other reaction products and scattered projectiles. The recoil detector spectrum is shown in Fig. 5, where a peak from ${ }^{14} \mathrm{~N}$ recoils was observed. The shaded spectrum was produced by gating on alpha recoils in the SIDAR which reduced the background significantly.

\section{CONCLUSIONS}

We have made stable beam measurements of reactions similar to the ones we will measure with a radioactive ${ }^{17} \mathrm{~F}$ beam. We have demonstrated our readiness to perform radioactive ion beam experiments and measured reaction cross sections which will contribute background to our radioactive beam experiments. Properties of resonances in ${ }^{18} \mathrm{~F}$ have been measured with the ${ }^{1} \mathrm{H}\left({ }^{17} \mathrm{O},{ }^{1} \mathrm{H}\right){ }^{17} \mathrm{O}$ reaction

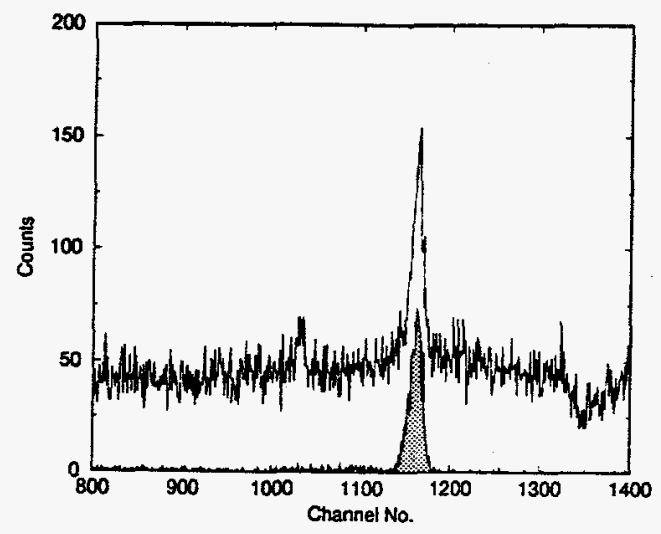

FIGURE 5. The energy spectrum from the recoil silicon detector. The shaded graph was gated on alphas in the SIDAR. using the SIDAR, and a measurement of the ${ }^{12} \mathrm{C}(\mathrm{p}, \gamma){ }^{13} \mathrm{~N}$ reaction cross section has been made using the DRS. We have, furthermore, made a measurement of the ${ }^{1} \mathrm{H}\left({ }^{17} \mathrm{O}, \alpha\right){ }^{14} \mathrm{~N}$ cross section by detecting ${ }^{14} \mathrm{~N}$ and alpha recoils in coincidence in two detector arrays.

\section{ACKNOWLEDGMENTS}

Many people have been involved in the DRS and SIDAR commissioning work. These include $P$. Bertone, J. C. Blackmon, A. E. Champagne, A. A. Chen, T. Davinson, U. Greife, V. Hansper, M. A. Hofstee, A. N. James, B. A. Johnson, P. E. Koehler, R. L. Kozub, Z. Ma, J. W. McConnell, W. T. Milner, P. D. Parker, D. E. Pierce, M. Rabban, D. Shapira, A. C. Shotter, M. S. Smith, F. Strieder, K. B. Swartz, C. Ulrey, D. W. Visser, and P. J. Woods. Research at the Oak Ridge National Laboratory is supported by the U. S. Department of Energy under contract DE-AC05-960R22464 with Lockheed Martin Energy Research Corp. Additional support is provided by contracts DE-FG02-91ER40609 with Yale University, and DE-FG02-96ER40995 with Tennessee Technological University.

\section{REFERENCES}

1. Wallace, R. K., and Woosley, S. E., Ap. J. Suppl. 45, 389-420 (1981).

2. Schatz, H., et al., Phys. Rep. 294, 168-263 (1998).

3. Garrett, J. D., et al., Nucl. Phys. A557, C701-C714 (1993).

4. Ball, J. B., Nucl. Phys. A570, C15-C22 (1994).

5. Alton, G. D., et al., Nucl. Inst. Meth. A328, 325-329 (1993).

6. Alton, G. D., et al., Nucl. Inst. Meth. A382, 207-224 (1996).

7. Alcen ${ }^{\text {TM }}$, Alumina Fiber, RATH Performance Fibers, Wilmington, DE, USA.

8. García, A., et al., Phys. Rev. C43, $2012-2019$ (1991).

9. Hahn, K. I., et al., Phys. Rev. C54, 1999-2013 (1996).

10.Park, S. H., et al., submitted to Phys. Rev. C.

11.Coszach, R., et al., Phys. Lett. B353, 184-188 (1995).

12.Blatt, J. M., and Biedenharn, L. C., Rev. Mod. Phys. 24, 258-272 (1952).

13.Tilley, D. R., et al., Nucl. Phys. A595, 1-170 (1995).

14.James, A. N., et al., Nucl. Inst. Meth. A267, 144-152 (1988).

15.Smith, M. S., et al., Nucl. Inst. Meth. A306, 233-239 (1991).

16.Smith, M. S., "Nuclear Astrophysics and the Daresbury Recoil Separator at the Holifield Radioactive Ion Beam Facility," presented at the International Symposium on Origin of Matter and Evolution of Galaxies 97, Atami, Japan, Nov. 5-7, 1997. 